\title{
O ensino da filosofia no limiar da contemporaneidade. $O$ que faz o filósofo quando seu ofício é ser professor de filosofia? Rodrigo Pelloso Gelamo
}

\author{
Editora Cultura Acadêmica, Universidade Estadual Paulista \\ (UNESP), São Paulo, Brasil, 2009, 179 págs.
}

\section{Gonzalo Montenegro}

Universidad Estadual Paulista, Assis-SP, Brasil. Email: gozznl@gmail.com

Rodrigo Pelloso Gelamo es Doctor en Educación y Profesor de la Facultad de Filosofía y Ciencias de la Universidad Estadual Paulista (UNESP) en Marília-SP, Brasil. Su investigación ha estado abocada al estudio de la naturaleza de la enseñanza de la filosofía en conexión con los contextos históricos y políticos de formación del pensamiento filosófico y educativo brasileño.

La obra de Rodrigo Pelloso Gelamo se funda en la convicción de que los problemas prácticos comprometidos en la enseñanza de la filosofía corresponde tratarlos dentro del ámbito propiamente filosófico. El subtítulo del estudio O que faz o filósofo quando seu ofício é ser professor de filosofia? supone, en este sentido, que la tarea característica y desafíos del profesor se confunden con algunas de las matrices más relevantes del desarrollo de la historia de la filosofía. En efecto, la cuestión relativa a la enseñanza, la inquietud acerca del aprendizaje del pensamiento y las preguntas sobre la naturaleza del conocimiento filosóficos, constituyen algunas de las preguntas que, junto con determinar la labor del profesor encargado de esta disciplina, atraviesan una buena parte de sus cuestiones fundamentales.

Al mismo tiempo, Gelamo da cuenta panorámicamente de las razones históricas que motivan en Brasil el desconocimiento o desprecio por tales estudios al interior de la filosofía. El rigor argumentativo de la obra se evidencia en el cuidadoso seguimiento de los antecedentes histórico-filosóficos del problema planteado, así como en la inquietud por definir una base metodológica adecuada que establezca los deslindes necesarios respecto de los asuntos estudiados en el seno de la pedagogía o de la filosofía de la educación. De este modo, los resultados, abocados principalmente a pensar la experiencia del quehacer filosófico ofrecen un verdadero referente sobre la cual debiera desenvolverse en la actualidad el problema de la enseñanza de la filosofía. 
La obra consta de tres capítulos 1. O ensino da Filosofia: entre a questão pedagógica e a problemática filosófica, 2. O problema do ensino da Filosofia y 3. Experimentar pensar.

$\mathrm{Al}$ interior del primer capítulo, en la primera sección Apresentação do problema, se establecen las coordenadas del estudio en términos de los contextos que permiten situarlo. Junto con diagnosticar el estado de la cuestión en la historia de la filosofía brasileña, el estudio ofrece una verdadera genealogía del pensamiento filosófico brasileño. Ello demuestra que el posicionamiento ante el problema de su enseñanza juega un rol fundamental en el propio desarrollo general de la filosofía en Brasil, con lo cual se refuerza la tesis central del estudio con todavía más fuerza: las cuestiones relativas a la enseñanza de la filosofía conciernen a un problema esencialmente filosófico. Así se da cuenta, entre otros asuntos, de la tensión persistente entre las expectativas que traza el conocimiento práctico de la docencia y aquellas que surgen de la formación filosófica. También son abordadas las problemáticas que caracterizan el lugar de la filosofía en el seno de las formaciones universitarias más diversas. Ello contribuye a proporcionar un marco más preciso a la hora de ingresar en el análisis del estatuto de cursos como "Filosofía de la Educación", cuando estos se encuentran insertos en la carrera de formación de profesores de filosofía. Con ello Gelamo logra medir una de las confrontaciones que definen el problema relativo al quehacer del filósofo en cuanto profesor de filosofía. En efecto, según describe el autor, aquí se juega una de las constantes con las que tiene que lidiar la formación contemporánea del profesor de filosofía, cual es la escasa relación entre las teorías educativas, la historia de la filosofía de la educación, el ejercicio de la enseñanza de filosofía y el pensamiento filosófico tal como este se estudia en la tradición. El mencionado curso aparece como el lugar paradigmático en que estos elementos entran en un conflicto caracterizado por la mutua y generalizada indiferencia.

Otra de las confrontaciones que Gelamo identifica y describe, dice relación con el conflicto que separa el estudio de la historia de la filosofía, al que se consagran las formaciones de grado en filosofía, y la preparación para el ejercicio docente en filosofía, al que se consagran formaciones en paralelo o consecutivas donde entra en escena la tradición educativa. La historia ignora a la enseñanza, o bien, esta se define en franca contradicción con la historia. Aquí Gelamo ingresa en uno de las cuestiones clásicas relativas a la forma en que la filosofía enfrenta la cuestión de su enseñabilidad. Bajo el título $O$ ensino da Filosofia como problema filosófico (segunda sección del capítulo primero) aborda la cuestión ampliamente conocida relativa a las diferencias con que Kant y Hegel abordan el asunto. El decurso del capítulo primero coloca, pues, al lector paulatinamente en el territorio que permite ir desde la hesitación con que nuestra época enfrenta el asunto relativo a la enseñanza de la filosofía, tanto en la formación universitaria como en la escuela, hasta la identificación de los fundamentos con que la filosofía se ha hecho cargo tradicionalmente del asunto.

La entrada en el conflicto de Kant con Hegel no constituye, sin 
embargo, más que la apertura del ámbito de problematización adecuado para entrar en lo que interesa propiamente a Gelamo: la filosofía que impregna la enseñanza de la filosofía. El segundo capítulo se consagra in extenso al análisis de las coordenadas que podrían permitir una articulación adecuada a esta filosofía, que, como podemos apreciar, no descuida en modo alguno las cuestiones relativas a la docencia. Aquí el autor se apoya en las lúcidas propuestas que Deleuze y Foucault proporcionan respecto del pensar y la formación que este exige. La primera sección Pensar sem pressupostos realiza un balance general de la concepción de pensamiento que rige sobre buena parte de las prácticas docentes en filosofía. Apoyado principalmente en el Cap. III de Diferencia y repetición de Deleuze, Gelamo muestra la íntima relación existente entre formas típicas de la docencia escolar o universitaria y una determinada concepción de pensamiento que impide el libre cultivo de la filosofía, razón por la cual el francés denomina dicha concepción como imagen dogmática del pensar. Gelamo acompaña a Deleuze en esta crítica y pretendiendo posicionarla en el horizonte de una nueva propuesta respecto del asunto que le inquieta, se proyecta hasta la concepción de problematización de la actualidad que Foucault desarrolla durante los años 1980 bajo el concepto de ontología del presente (segunda sección: Uma atitude diante dos problemas: a ontologia do presente). Luego, en la tercera sección titulada $O$ lugar do conhecimento e da experiência no ensino da Filosofia, realiza un diálogo con las principales perspectivas que pueblan la discusión contemporánea sobre el modo de articular la tradición filosófica con la enseñanza. Concurren a enriquecer este diálogo estudiosos como Pagni, Bárcena, Larrosa, Rancière, Chauí y Benjamin.

El tercer capítulo, Experimentar pensar, transforma la mirada crítica de Deleuze y Foucault en una aproximación creadora capaz de posicionar las coordenadas principales de un ejercicio de pensamiento que se concibe en la formación. Formar y formar-se en el pensar aparecen como imperativos que borran no sólo la carga de la infinidad de dispositivos que aseguran una tradición de pensamiento dogmática, sino también las barreras que separan al profesor y al alumno, al saber del aprendizaje. Uno de los efectos más impactantes de este capítulo radica en que piensa la cuestión relativa a los modos de subjetivación propuestos por Deleuze-Guattari, al mismo tiempo que la estética de la existencia foucaultiana, al margen de las coordenadas que nos obligan a realizar el trazado habitual que separa al educador del educando. Como destaca Gelamo el pensar instala un campo de inmanencia cuya exigencia de experimentar en la creación de nuevos modos de existencia resulta indiferente respecto de las coordenadas clásicas conforme se piensa la formación. Si pensar consiste en engendrar el pensamiento dentro de sí y con ello formarse, ¿habrá alguna diferencia substantiva entre el profesor, el filósofo y el estudiante? Ante esta pregunta desafiante nos deparamos a medida que recorremos este último capítulo y sus conclusiones.

La pertinencia y actualidad del estudio resulta innegable para nuestro contexto latinoamericano. Las transformaciones que la región sufre en los más diversos ámbitos y la necesidad de confrontar con coraje la invención del futuro penetra en la filigrana de la conformación de los saberes y 
cuestiona, sin duda, el lugar de la filosofía y el papel que cabe a sus respectivos profesores. La región, sabemos, enfrenta una fiebre de transformaciones curriculares en más diversos niveles del sistema educativo, las que se enfrentan a la gran encrucijada de responder a las presiones de la transformación del sistema universitario europeo (Proceso de Bolonia, Proyecto Tuning), al mismo tiempo que profundizar los procesos de democratización todavía necesarios en una región cuyos sistemas educativos todavía arrastran el lastre de las dictaduras que desangraron el continente durante la segunda mitad del siglo XX. Esta encrucijada alcanza en la actualidad un punto álgido. En este contexto, el re-posicionamiento curricular de la filosofía en la enseñanza secundaria exige afrontar reflexiones como las que lleva adelante esta investigación, la que nos invita a pensar e inventar estas nuevas formas de pensar hoy la formación filosófica.

Es conveniente destacar, en último término, que el riguroso deslinde realizado respecto de la pedagogía o de la filosofía de la educación a lo largo del estudio -en tanto áreas probablemente idóneas para hacerse cargo de la tesis central de la obra y que Gelamo descarta ya dentro de las primera páginas del capítulo primero- refleja una serie de condicionantes propias de la historia y estructura curricular en que se insertan estas disciplinas en Brasil. En efecto, la Pedagogía en este país designa la formación relativa a los docentes de la Escuela Primaria. Los saberes y problemáticas que pueblan esta área de la educación se desarrollan, además, al alero del impulso de los procesos de socialización y civilización de la población que impregnan al positivismo brasilero desde comienzos de la República. La "Filosofía de la Educación”, por su parte, adolece de una pobreza conceptual derivada principalmente de la falta de problemáticas propias. Esta aparece más como una colección de opiniones sobre la educación desarrollada por filósofos diversos, que una confrontación de conceptos y cuestiones que podrían definir las matrices del fenómeno educativo. Lo anterior explica la decisión categórica con que Gelamo se niega a pensar la problemática central de su estudio a partir de las coordenadas proporcionadas por tales dominios. Esta negativa, con todo, no es en modo alguno individual y expresa la peculiaridad con que en Brasil se ha instalado paulatinamente el dominio de la filosofía de la enseñanza de la filosofía, radicado en facultades de educación y de humanidades, y confrontando a menudo escaramuzas diversas con los dos dominios anteriormente descritos. En Chile y Argentina, por mencionar realidades que nos resultan más conocidas, la tensión anterior no siempre se resuelve de igual modo. Así, por ejemplo, persiste una línea de desarrollo de la pedagogía que, en colaboración con la filosofía, ha asumido de modo atractivo algunos de los asuntos estudiados por Gelamo a lo largo de su obra. Esto, por cierto, a condición de comprender la pedagogía como una derivación histórica de la filosofía y las humanidades, y no como una ciencia o agrupación de ciencias próximas al fenómeno educacional. Concepción, la segunda, que pugna fuertemente por dominar el desarrollo y la arquitectura político-epistemológica de las facultades de educación. Ello explica el hecho de que las carreras que preparan profesores de filosofía, en Chile y Argentina, se denominen justamente 'Pedagogías'. Situación que no coincide con el contexto brasileño donde el término está 
reservado, como decíamos, exclusivamente a la formación de profesores de Educación Primaria, cuya cercanía histórica a los dispositivos de socialización y civilización de la población, los ha limitado en el acceso a las diversas cuestiones que inquietan a las disciplinas científicas o humanistas. De acuerdo con lo anterior el estudio de Gelamo proporciona también indicaciones interesantes a la hora de emprender estudios comparados respecto de cómo la formación del profesor de filosofía se desarrolla en la región. 